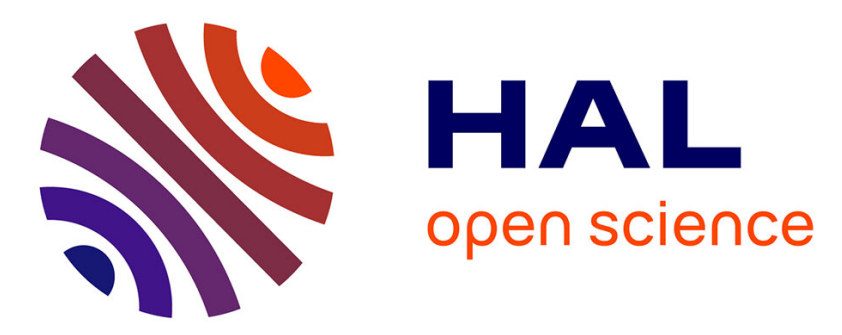

\title{
Le logiciel FREEL : réalisations en technologie hybride et monolithique d'amplificateurs microondes large bande pour dispositif de réception numérique sur fibre optique à $4,8 \mathrm{Gbit} / \mathrm{s}$ \\ P. Jarry, R. A. Soares, A. Perennec
}

\section{To cite this version:}

P. Jarry, R. A. Soares, A. Perennec. Le logiciel FREEL: réalisations en technologie hybride et monolithique d'amplificateurs microondes large bande pour dispositif de réception numérique sur fibre optique à 4,8 Gbit/s. Revue de Physique Appliquée, 1989, 24 (5), pp.529-537.

10.1051/rphysap:01989002405052900 . jpa-00246078

\section{HAL Id: jpa-00246078 \\ https://hal.science/jpa-00246078}

Submitted on 1 Jan 1989

HAL is a multi-disciplinary open access archive for the deposit and dissemination of scientific research documents, whether they are published or not. The documents may come from teaching and research institutions in France or abroad, or from public or private research centers.
L'archive ouverte pluridisciplinaire HAL, est destinée au dépôt et à la diffusion de documents scientifiques de niveau recherche, publiés ou non, émanant des établissements d'enseignement et de recherche français ou étrangers, des laboratoires publics ou privés. 


\title{
Le logiciel FREEL : réalisations en technologie hybride et monolithique d'amplificateurs microondes large bande pour dispositif de réception numérique sur fibre optique à $\mathbf{4 , 8} \mathrm{Gbit} / \mathrm{s}$
}

\author{
P. Jarry $\left({ }^{1}\right)$, R. Soares $\left({ }^{2}\right)$ et A. Perennec $\left({ }^{1}\right)$ \\ ( $\left.{ }^{1}\right)$ LEST, URA CNRS n 1329, Université de Brest, 6 av. Le Gorgeu, 29287 Brest Cedex, France \\ ( ${ }^{2}$ CNET-LAB/MER, B.P. 40, 22301 Lannion Cedex, France
}

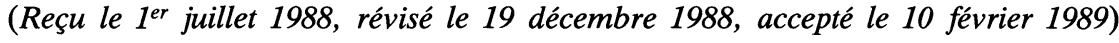

\begin{abstract}
Résumé. - Dans les systèmes à haut débit sur fibre optique il est nécessaire de disposer en réception d'un amplificateur ultra large bande à haut gain et bas niveau de bruit. Ce papier a pour but de montrer comment une technique analytique puissante, comme la méthode des fréquences réelles, peut être appliquée à la conception d'un amplificateur $6 \mathrm{MHz}-6 \mathrm{GHz}$. En accord avec cette théorie deux réalisations ont été faites, une en monolithique (amplificateur à deux étages avec $17 \mathrm{~dB}$ de gain) et une en hybride (amplificateur à trois étages et $16 \mathrm{~dB}$ de gain).
\end{abstract}

\begin{abstract}
In fiber optic very high datarate systems it is necessary to have an ultra large band high gain low noise amplifier after the front-end. The purpose of this paper is to show how powerful analytical techniques, such as the real frequency technique, may be applied to the design of a $6 \mathrm{MHz}-6 \mathrm{GHz}$ amplifier. According to the theory a two stage monolithique amplifier gives $17 \mathrm{~dB}$ gain and a 3 stage hybrid amplifier $16 \mathrm{~dB}$ across the band.
\end{abstract}

\section{Introduction.}

La technique des fréquences réelles $[1,2]$ n'a été appliquée jusqu'à présent qu'à la conception d'amplificateurs à deux ou trois octaves de bande passante. Dans les systèmes de réception à haut débit sur fibre optique, il est nécessaire de couvrir des bandes passantes ayant des largeurs typiques de trois décades. Ainsi un système optique à $4,8 \mathrm{Gbit} / \mathrm{s}$ nécessite une amplification sur une bande de $6 \mathrm{MHz}$ à $6 \mathrm{GHz}$.

Jusqu'à présent, la conception d'amplificateurs multi-décades était basée sur des principes empiriques. Dans cet article nous appliquons la technique des fréquences réelles et le logiciel associé FREEL (FREquences REELles, élaboré au LEST) pour concevoir un amplificateur $6 \mathrm{MHz}-6 \mathrm{GHz}$.

La technique est appliquée pour optimiser, à l'aide de l'algorithme de Moré $[4,5]$, les valeurs des éléments des égaliseurs du type passe-bas placés entre les «blocs» que constitue chaque transistor associé à son circuit de contre-réaction et de polarisation. L'utilisation du progiciel Acline a permis de prendre en compte les plots de connection nécessaires à l'implantation. Deux technologies de réalisation de l'amplificateur ont été étudiées. Une en monolithique à deux étages réalisée à la fonderie de Plessey III-V, et un amplificateur hybride à trois étages. Le gain, le facteur de bruit et les réponses temporelles sont présentées pour chaque amplificateur.

\section{Technique de synthèse.}

Nous avons présenté dans une précédente communication [5] une méthode originale dite des fréquences réelles qui permet de concevoir des amplificateurs à plusieurs étages. Le caractère itératif de la méthode permet d'optimiser de manière simple le gain et le TOS de l'ensemble multi-étages. Cette méthode, pour être étendue à la conception d'amplificateurs très large bande, nécessite l'utilisation d'éléments résistifs en particulier au niveau des circuits de contre-réaction pour assurer une bonne stabilité. Il est donc nécessaire de définir une nouvelle topologie des transistors FET utilisés.

TOPOlOGiE. - Aux basses fréquences microondes un transistor MESFET AsGa est instable et il est virtuellement impossible de l'adapter sur de très 
larges bandes en utilisant des réseaux constitués uniquement d'éléments passifs. Ceci est dû principalement à la valeur importante du coefficient de réflexion à l'entrée et au gain intrinsèque élevé du transistor. Il est donc indispensable d'utiliser des éléments résistifs. Ils seront disposés d'une part en parallèle avec la grille, et d'autre part, dans un circuit RLC de contre-réaction. Leur action a pour but de faire chuter le gain aux basses fréquences et d'adapter l'entrée du transistor. Dans un deuxième temps nous considérerons les circuits de polarisation. On constitue ainsi un ensemble que nous appellerons Transistor Feedback Block (TFB), caractérisé par des nouveaux paramètres de répartition. La technique des fréquences réelles est donc appliquée aux blocs (TFB).

OPTIMISATION DU GAIN ET DU TOS [4, 5]. - La technique des fréquences réelles introduites par Carlin [1] est une approche optimum des problèmes d'adaptation à large bande. Cette technique a été étendue aux doubles adaptations et appliquée aux amplificateurs multi-étages [2]. Dans cette partie, nous montrons comment la technique des fréquences réelles pour l'adaptation des amplificateurs multiétages comportant des TFB peut aussi être utilisée pour une optimisation simultanée gain-TOS. L'algorithme d'optimisation est celui de Moré $[4,5]$. C'est une amélioration de la méthode de Levenberg-Marquardt. Le critère utilisé est celui des moindres carrés.

Prenons la figure 1 , pour les $k$ premiers étages de l'amplificateur, le gain transducique est donné par [2] :

$$
T_{k}(\omega)=T_{k-1} \frac{\left|e_{21 k}\right|^{2}\left|S_{21 k}\right|^{2}}{\left|1-e_{11 k} S_{G k}\right|^{2}\left|1-\hat{e}_{22 k} S_{11 k}\right|^{2}}
$$

c'est-à-dire :

$$
T_{k}(\omega)=T_{k-1}, E_{k}(\omega)
$$

où :

$T_{k-1}$ : désigne le gain des $(k-1)$ premiers étages $\left(e_{i j}\right)_{k}$ : paramètres de répartition du $k$-ième égaliseur $E_{k}$

$\left(S_{i j}\right)_{k}$ : paramètres de répartition du $k$-ième TFB.

Le gain en puissance totale $T(\omega)$ est défini lorsque le dernier égaliseur $E_{k+1}$ ferme le réseau :

$$
T(\omega)=\left(T_{1} \cdot T_{2} \ldots T_{k}\right) E_{(k+1)}(\omega) .
$$

De même nous pouvons donner l'expression du taux d'ondes stationnaires de l'amplificateur multiétages. Conformément à la figure 1 , le TOS d'entrée au niveau du $k$-ième étage est :

$$
R_{\text {in } k}=\left.\frac{1+\left|\hat{e}_{111}\right|}{1-\left|\hat{e}_{111}\right|}\right|_{k}
$$

$R_{\text {in } k}$ est calculé de manière récursive à partir de la charge et son optimisation se fait parallèlement à celle du gain.

Après avoir ajouté le dernier égaliseur $E_{k+1}$ le TOS de sortie est donné par:

$$
R_{\text {out }}=\frac{1+\left|\hat{e}_{22 k+1}\right|}{1-\left|\hat{e}_{22 k+1}\right|} .
$$

Il a été montré [3] que les paramètres de répartition de l'égaliseur $E_{k}$ sont entièrement déterminés à partir de la connaissance d'un seul polynôme noté $h(p)$. En effet $E_{k}$ est un quadripôle réciproque et sans pertes, ses paramètres sont alors donnés par :

$$
\begin{aligned}
& e_{11 k}(p)=\frac{h(p)}{g(p)} \\
& e_{12 k}(p)=e_{21 k}(p)= \pm \frac{p^{\ell}}{g(p)} \\
& e_{22 k}(p)=-(-1)^{\ell} \frac{h(-p)}{g(p)} .
\end{aligned}
$$

Les composantes de $h(p)=h_{0}+h_{1} p+\cdots+h_{n} p^{n}$ seront les inconnues et $g(p)$ sera déterminé après factorisation de la relation de non-dissipativité de $E_{k}$ :

$$
g(p) g(-p)=h(p) h(-p)+(-1)^{\ell} p^{2 \ell}
$$

$\ell$ désigne l'ordre des zéros de transmission.

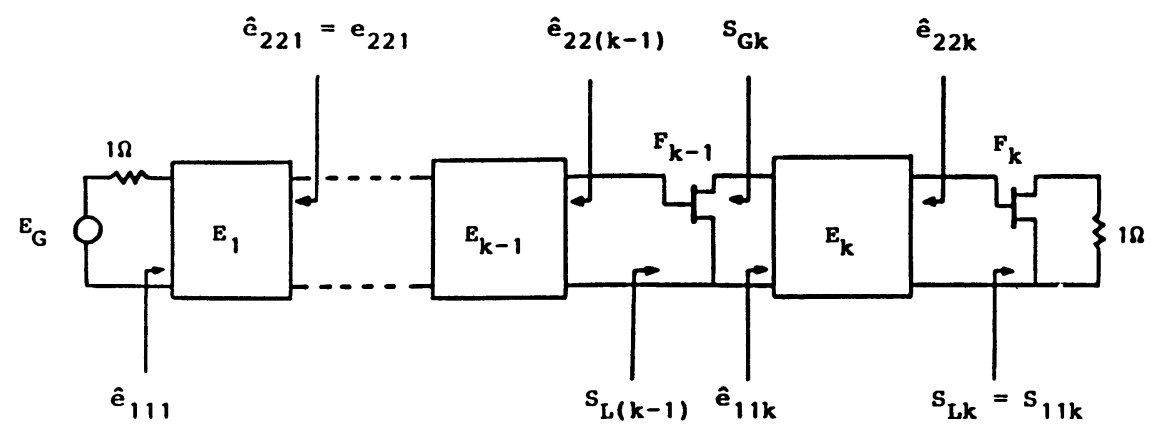

Fig. 1. - Schéma de l'amplificateur multi-étages.

[Broad-band multistage microwave amplifiers.] 
Le gain en puissance et le TOS d'entrée sont optimisés simultanément étage par étage en minimisant la fonction objectif suivante :

$$
\begin{aligned}
E^{2}=\sum_{j=1}^{m}\left\{W _ { 1 } \left(T\left(\omega_{j}\right) / T_{0}\right.\right. & -1)^{2}+ \\
& \left.+W_{2}\left(R_{\text {in }}\left(\omega_{j}\right) / R_{\text {in } 0}-1\right)^{2}\right\}
\end{aligned}
$$

$W_{1}$ et $W_{2}$ étant des poids affectés aux deux types d'erreurs relatives. Une des particularités de cette méthode est sa très bonne convergence ; elle est en effet assurée quel que soit le vecteur initial. Ce logiciel, disponible au LEST, permet également de concevoir des amplificateurs comportant des égaliseurs passe-bande $(\ell \neq 0)$.

\section{Réalisation hybride $[5,6,8]$.}

La technique des fréquences réelles a été appliquée à la conception d'un amplificateur $6 \mathrm{MHz}-6 \mathrm{GHz}$. La conception s'est déroulée en suivant toutes les étapes décrites plus haut. Les éléments des circuits de contre-réaction et de polarisation sont fixés par l'utilisateur avant l'optimisation des égaliseurs. Les selfs de connexion du transistor sont également prises en compte. On peut voir sur la figure 2 le

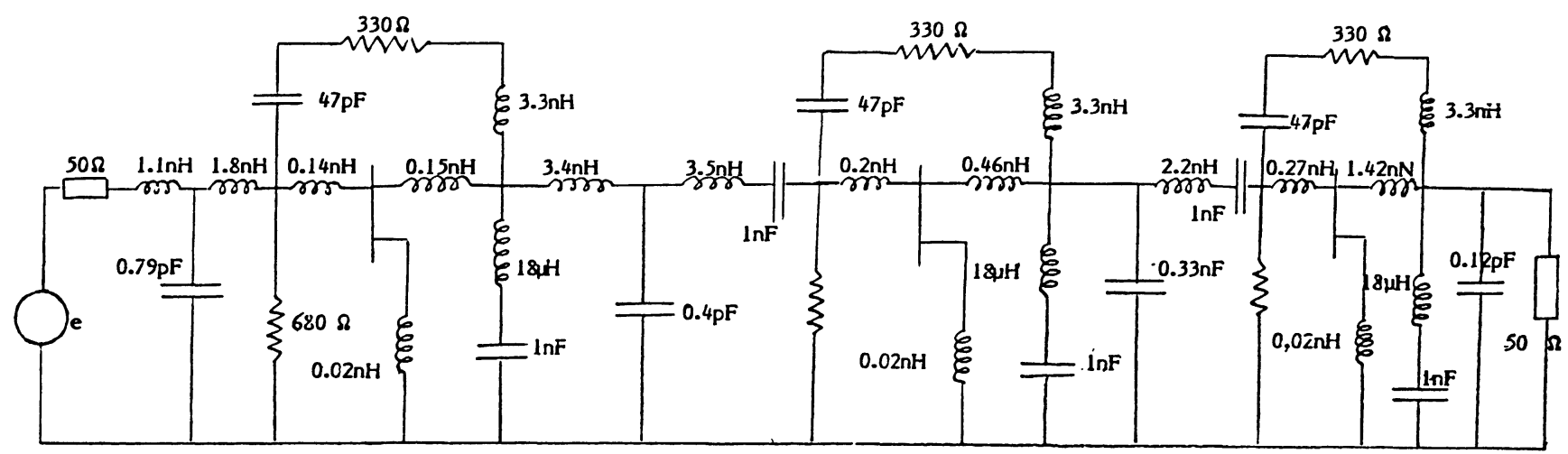

Fig. 2. - Amplificateur très large bande (6 MHz-6 GHz).

[Broad-band amplifier $(6 \mathrm{MHz}-6 \mathrm{GHz})$.]

Tableau I. - Paramètre $S d u C F X 31 X$ et du TFB.

[S parameters of CFX31X and TFB.]

Paramètres $S$ du CFX31X

\begin{tabular}{lllllllll} 
FREQ & \multicolumn{2}{c}{$S_{11}$} & \multicolumn{2}{c}{$S_{12}$} & \multicolumn{2}{c}{$S_{21}$} & \multicolumn{2}{c}{$S_{22}$} \\
$(\mathrm{GHz})$ & MOD & PHASE & MOD & PHASE & MOD & PHASE & MOD & PHASE \\
0,05 & 0,999 & $-1,4$ & 0,001 & 89,2 & 3,87 & 179 & 0,660 & $-0,5$ \\
0,9 & 0,989 & $-25,1$ & 0,021 & 76,1 & 3,77 & 162 & 0,650 & $-9,19$ \\
1,75 & 0,956 & $-48,4$ & 0,04 & 62,6 & 3,5 & 145 & 0,631 & $-18,9$ \\
2,6 & 0,892 & -69 & 0,056 & 50 & 3,68 & 127 & 0,600 & $-30,8$ \\
3,45 & 0,871 & $-85,1$ & 0,064 & 31,5 & 2,71 & 115 & 0,579 & $-38,5$ \\
4,3 & 0,871 & $-99,8$ & 0,069 & 34,6 & 2,39 & 103 & 0,569 & $-44,8$ \\
5,15 & 0,826 & -111 & 0,072 & 30,1 & 2,12 & 92,5 & 0,570 & $-49,9$ \\
6 & 0,810 & -120 & 0,073 & 27,0 & 1,92 & 85 & 0,570 & $-54,0$
\end{tabular}

Paramètres $S$ du TFB

\begin{tabular}{llllrcccc} 
FREQ & \multicolumn{2}{c}{$S_{11}$} & \multicolumn{2}{c}{$S_{12}$} & \multicolumn{2}{c}{$S_{21}$} & \multicolumn{2}{c}{$S_{22}$} \\
$(\mathrm{GHz})$ & MOD & PHASE & MOD & PHASE & MOD & PHASE & MOD & PHASE \\
0,05 & 0,376 & $-11,7$ & 0,151 & 7,49 & 2,22 & 175 & 0,242 & $-11,7$ \\
0,9 & 0,374 & $-25,7$ & 0,150 & $-6,17$ & 2,18 & 168 & 0,234 & 1,65 \\
1,75 & 0,405 & $-48,5$ & 0,144 & $-12,5$ & 2,17 & 155 & 0,250 & 0,86 \\
2,6 & 0,440 & $-68,9$ & 0,133 & $-17,9$ & 2,12 & 140 & 0,271 & $-6,7$ \\
3,45 & 0,500 & $-85,7$ & 0,121 & $-23,2$ & 2,06 & 128 & 0,296 & $-12,7$ \\
4,3 & 0,562 & -102 & 0,106 & $-26,7$ & 2,00 & 115 & 0,330 & $-19,3$ \\
5,15 & 0,605 & -115 & 0,092 & $-27,4$ & 1,91 & 102 & 0,369 & $-26,5$ \\
6 & 0,641 & -127 & 0,079 & $-25,5$ & 1,83 & 91,5 & 0,396 & $-33,2$
\end{tabular}


circuit final. Chaque égaliseur comportait au départ trois éléments, cependant si le résultat de l'optimisation donne des éléments dont les valeurs sont jugées trop petites, ceux-ci sont supprimés avant une nouvelle optimisation. A noter que des capacités de liaison de forte valeur $(100 \mathrm{nF})$ ont été rajoutées au niveau des lignes $50 \Omega$.
Avant l'implantation, le circuit a été repris sur le progiciel Acline en rajoutant les plots de connexion et les selfs de liaison des résistances de grille. Une réoptimisation de l'ensemble a été alors effectuée, donnant des réponses similaires.

L'amplificateur a été réalisé au CNET de Lannion B. Les transistors utilisés sont des CFX31X AsGa de

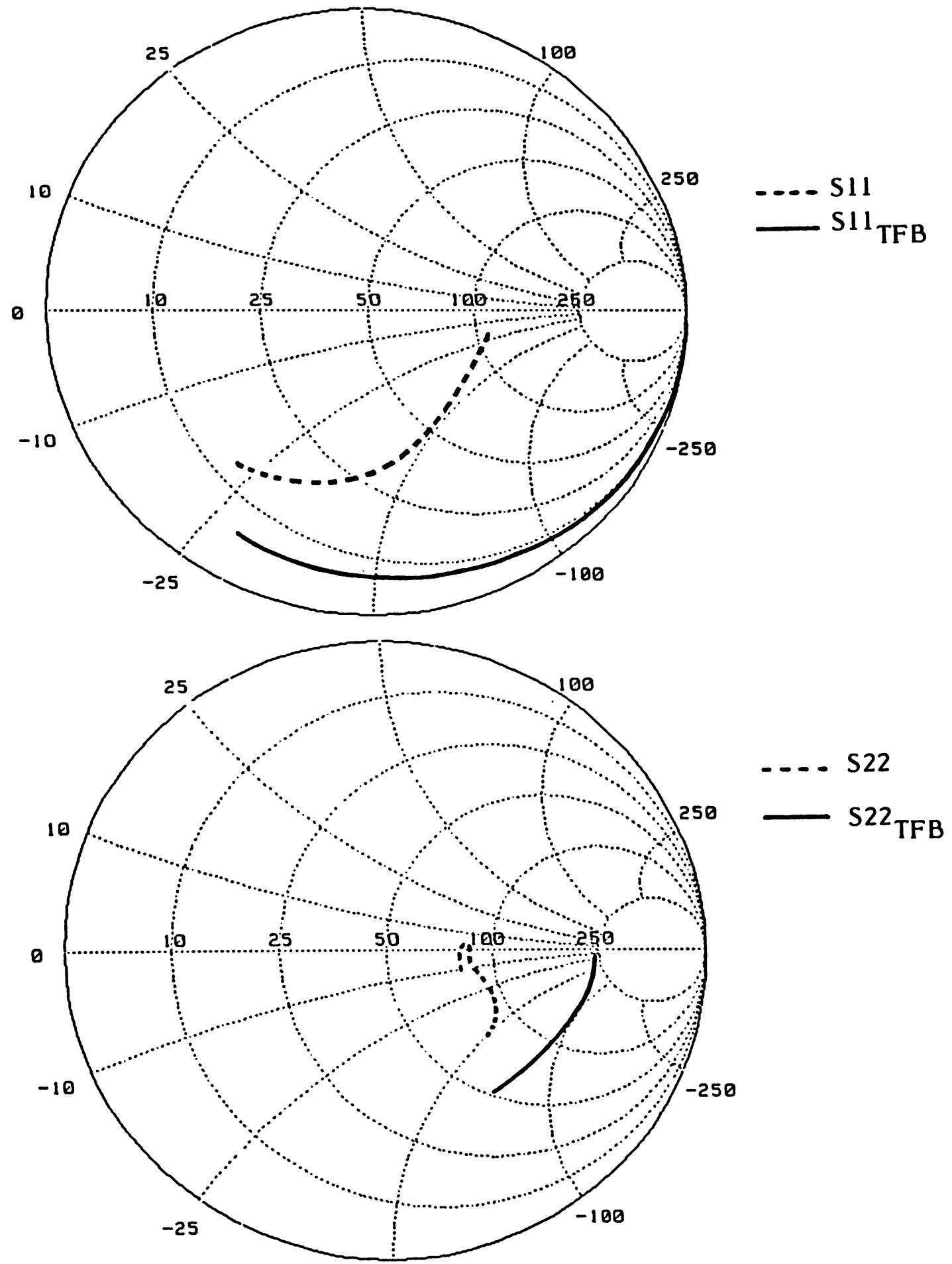

Fig. 3. - Paramètres $S$ du CFX31X et du TFB.

[Scattering parameters of CFX31X and TFB.] 


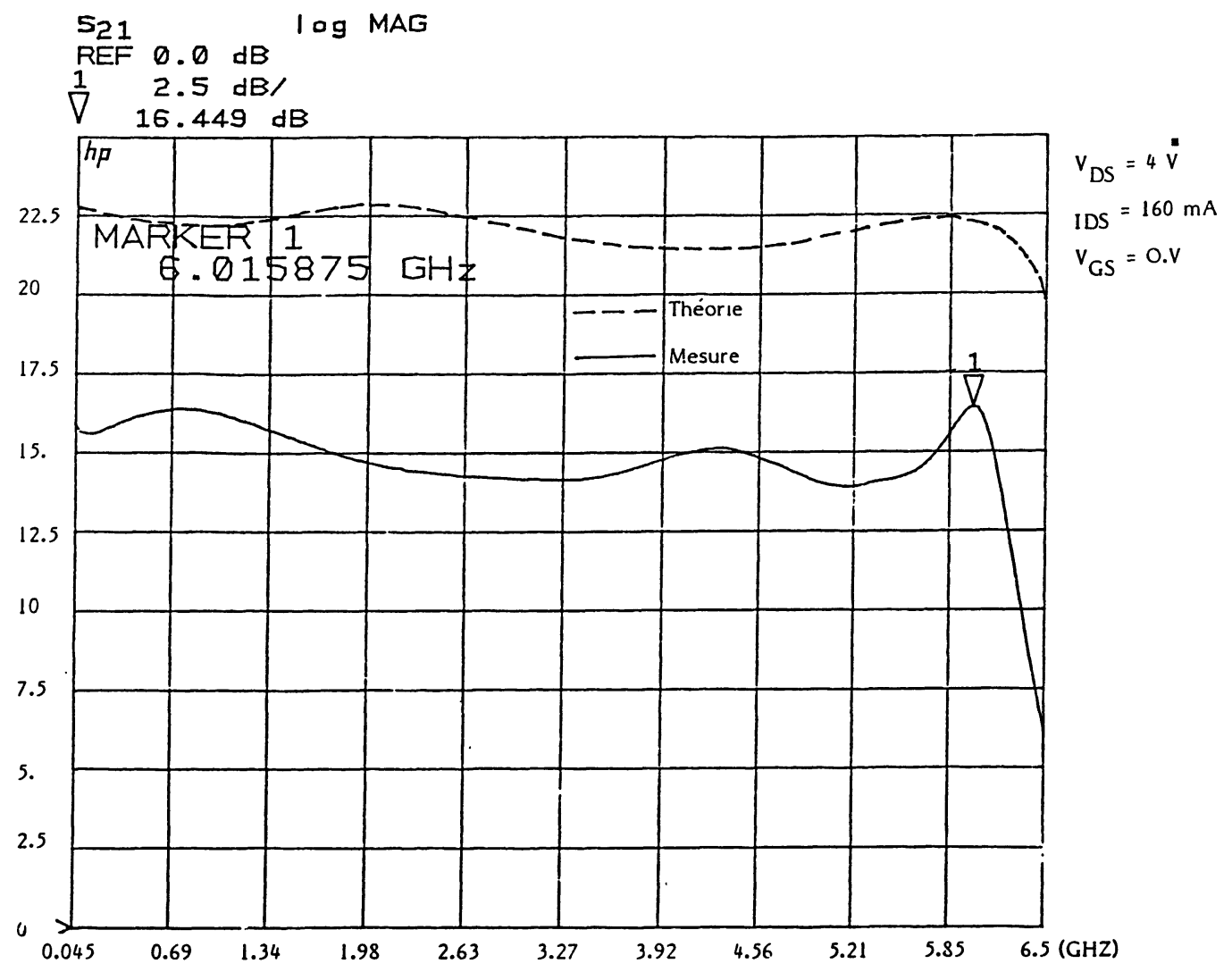

Fig. 4. - Courbes de gain théorique et mesurée.

[Gain responses (theoretical and measured).]

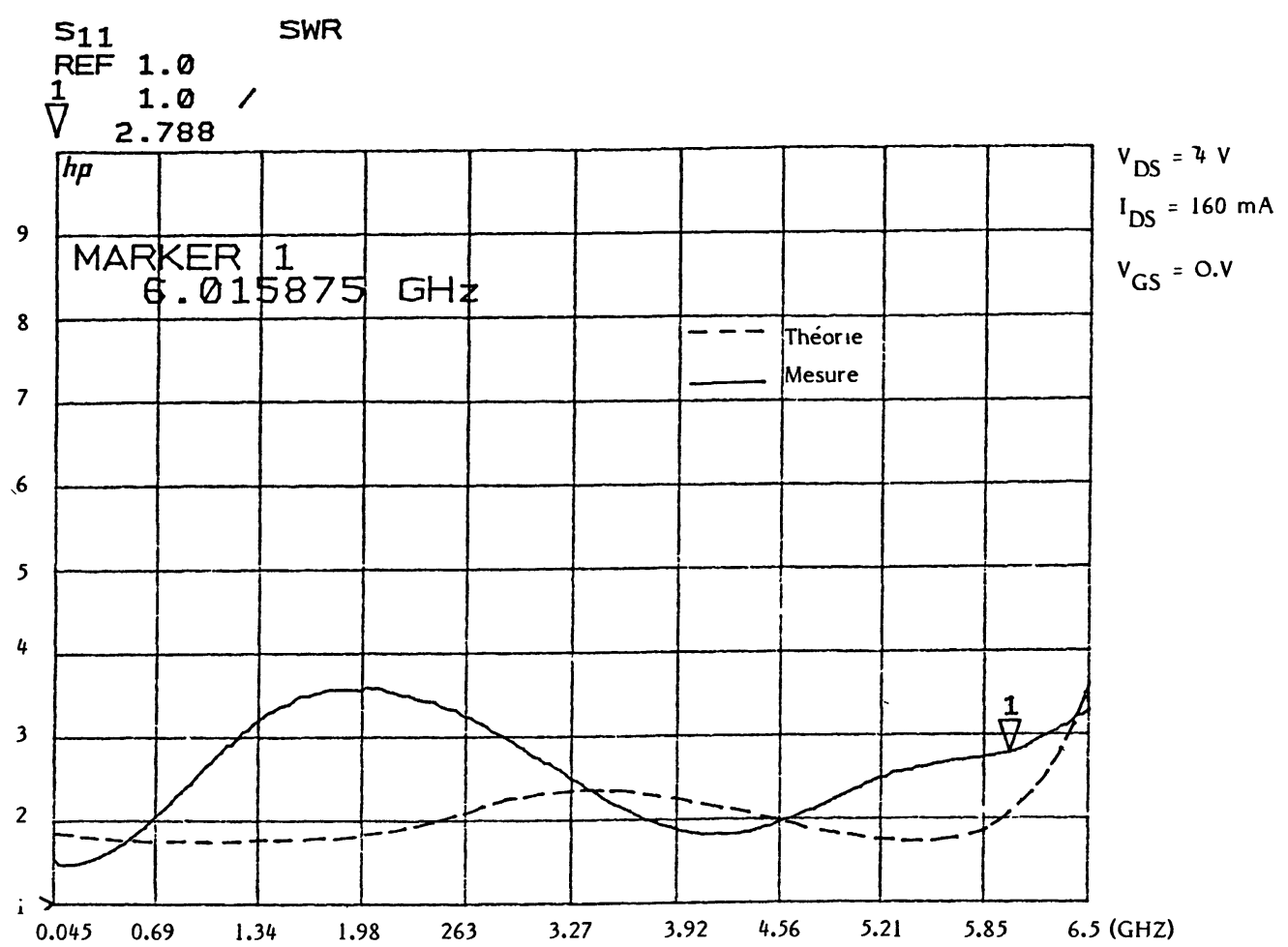

Fig. 5. - Courbes de TOS d'entrée théorique et mesurée.

[VSWR in (theoretical and measured).] 


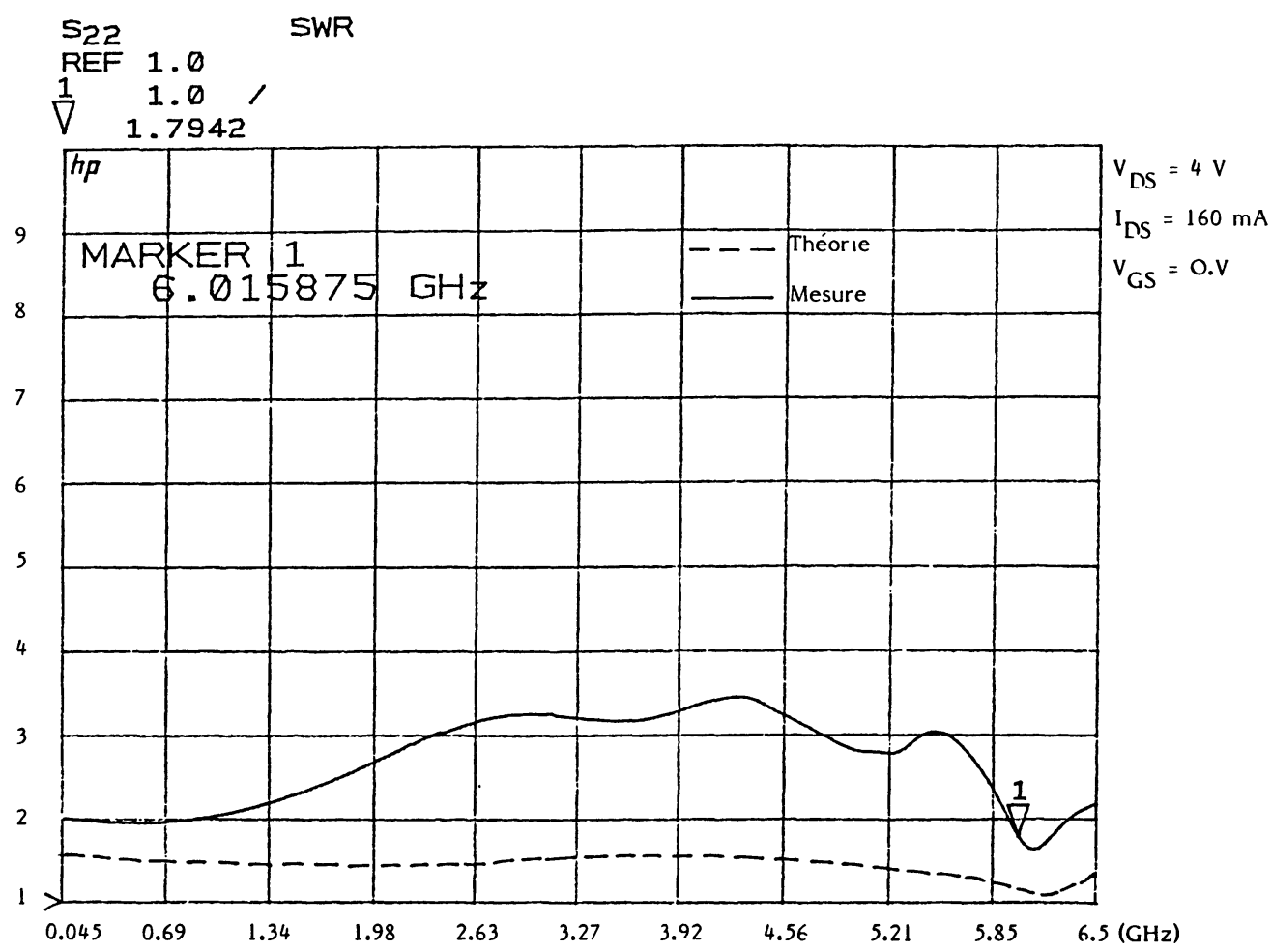

Fig. 6. - Courbes de TOS de sortie théorique et mesurée.

[VSWR out (theoretical and measured).]

RTC dont les paramètres $S$ des transistors seuls et après adjonction des éléments de contre-réaction et de polarisation sont présentés sur le tableau I et la figure 3. Seul le drain est alimenté $(4 \mathrm{~V})$. Le substrat servant à l'implantation est de l'alumine de un pouce carré et de $635 \mu \mathrm{m}$ d'épaisseur. Une amplification autour de $20 \mathrm{~dB}$ était souhaitée. Malgré des pertes par effet capacitif au niveau de la contre-réaction, donnant lieu à des réglages, un gain avec une bonne planéité autour de 15,5 dB a été obtenu (Fig. 4). La bande passante est entièrement couverte avec des TOS inférieurs à 3 (Figs. 5, 6). La puissance de sortie de $1 \mathrm{~dB}$ de compression est de $9 \mathrm{dBm}$. La photographie de l'amplificateur est donnée figure 7 . Le facteur de bruit mesuré a une valeur moyenne de $9.5 \mathrm{~dB}$

La figure 8 représente la réponse de l'amplificateur à un signal carré de fréquence $5 \mathrm{Gbit} / \mathrm{s}$. On observe que la distorsion est négligeable.

Ce premier exemple montre la validité de la méthode décrite. A noter que le caractère non unilatéral des transistors est pris en compte. Les courbes expérimentales sont en bon accord avec les résultats théoriques.

\section{Réalisation monolithique [6, 7].}

Le circuit de l'amplificateur monolithique a été réalisé à la fonderie de Plessey. A partir des schémas équivalents modélisant chaque élément du circuit

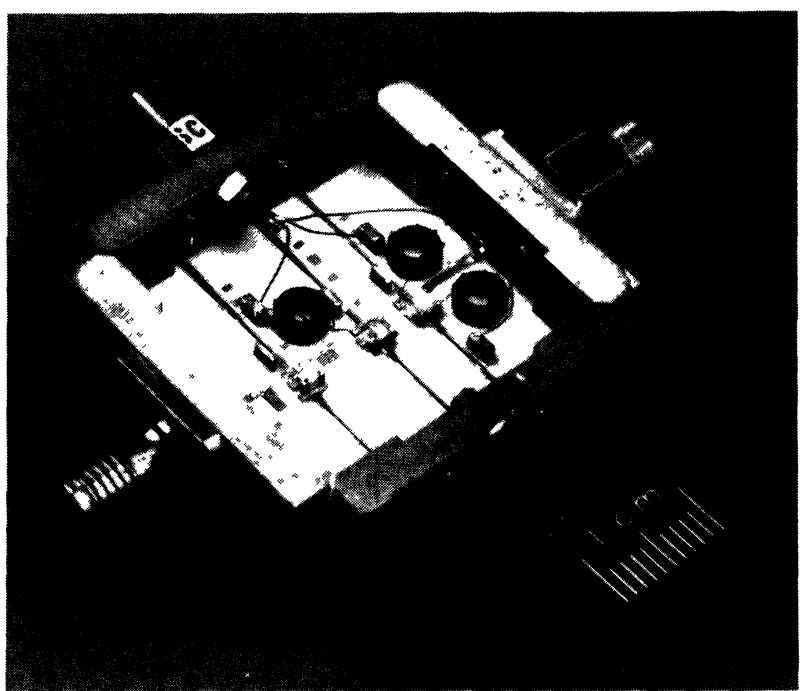

Fig. 7. - Réalisation hybride de l'amplificateur $6 \mathrm{Mhz}$ $6 \mathrm{GHz}$.

[Hybrid realization of $6 \mathrm{MHz}-6 \mathrm{GHz}$ amplifier.]

incluant les pertes, et des valeurs numériques données par la technique des fréquences réelles, une réoptimisation du circuit a été ensuite effectuée avec le progiciel Acline.

Les courbes 9 et 10 donnent les paramètres de répartition mesurés de l'amplificateur monolithique. On remarque que :

- le gain présente une bonne planéité autour de 


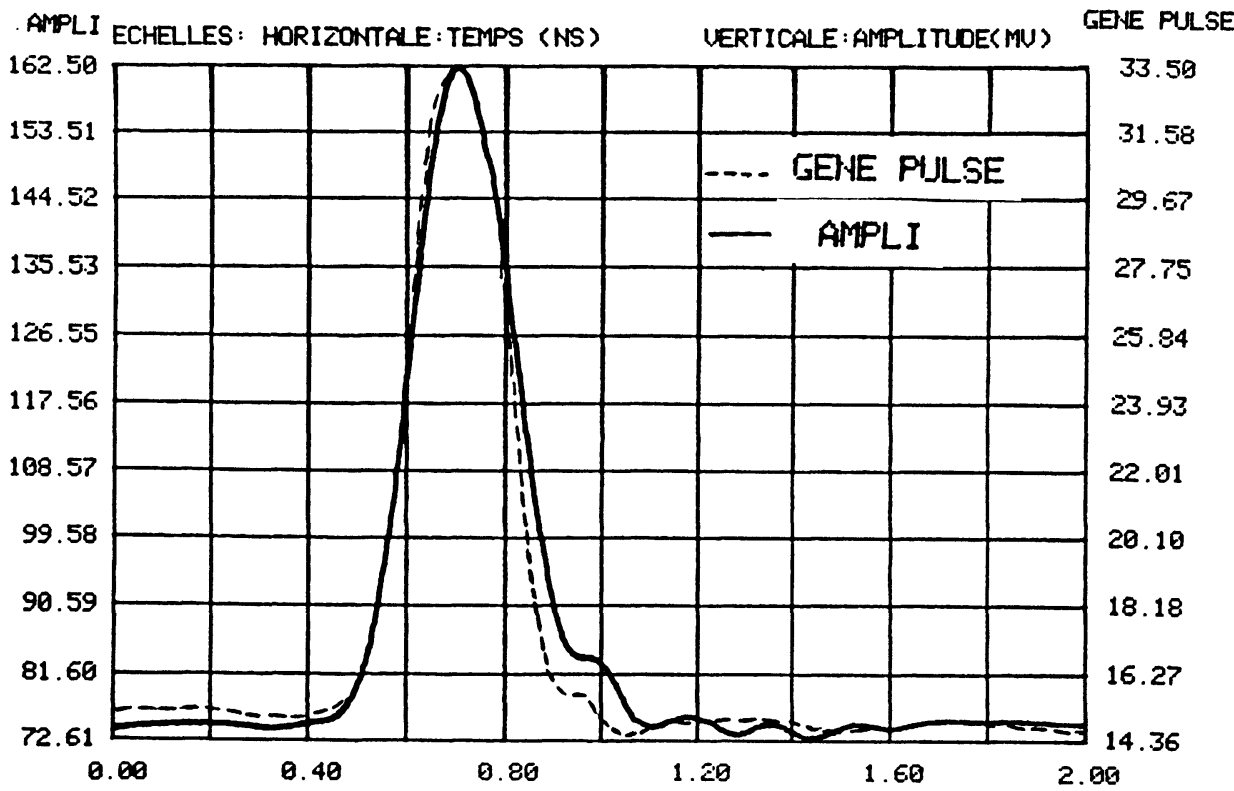

Fig. 8. - Réponses temporelles de l'amplificateur hybride.

[Temporal response of hybrid amplifier.]

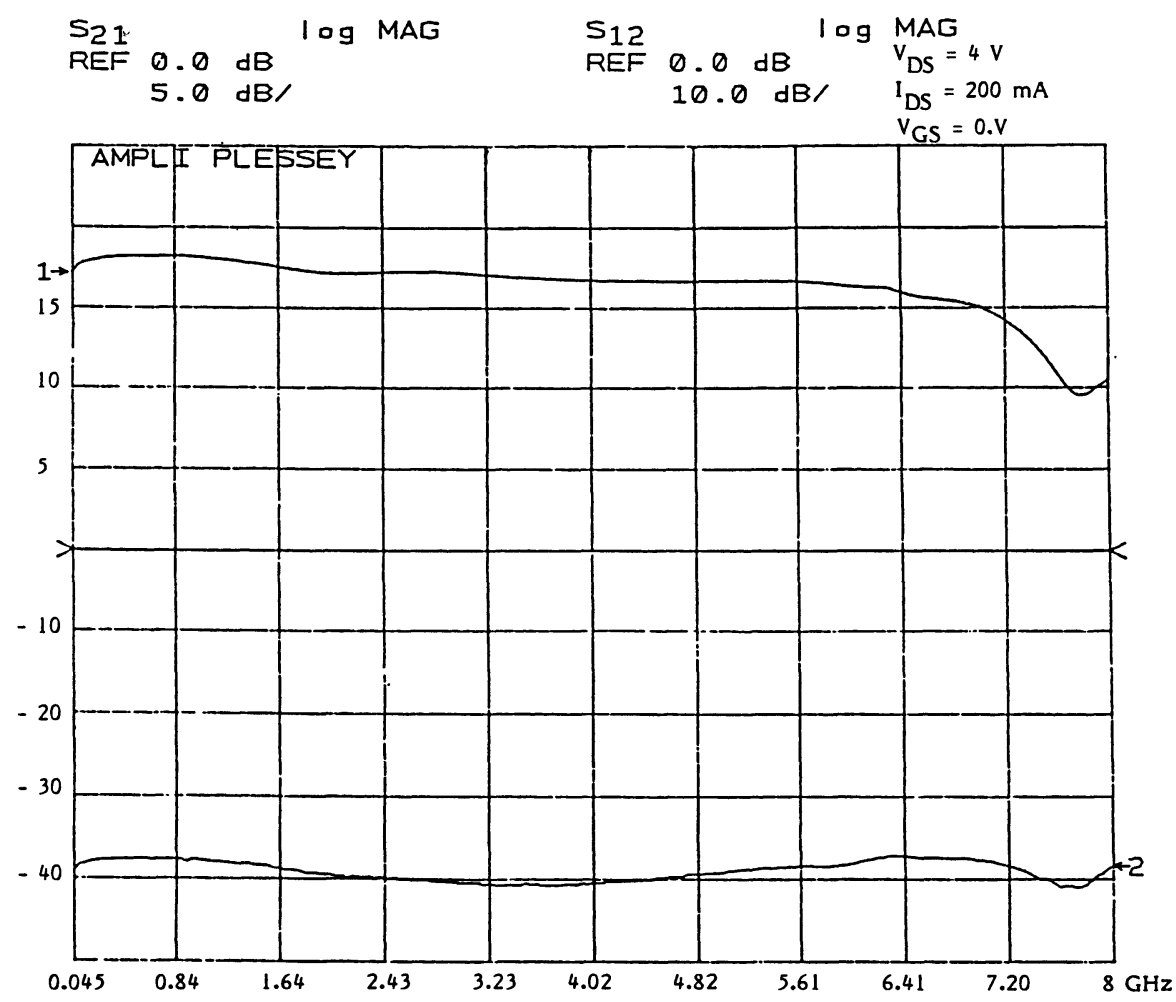

Fig. 9. - Courbes de gain et de $S_{12}$ de l'amplificateur monolithique.

[Gain response and $S_{12}$ of monolithic amplifier.]

$17 \mathrm{~dB}$ et est encore de $15 \mathrm{~dB}$ à $7 \mathrm{GHz}$. La fréquence de coupure basse est de $4 \mathrm{MHz}$;

- les pertes par réflexion sont inférieures à $-10 \mathrm{~dB}$.
La puissance de sortie de $1 \mathrm{~dB}$ de compression est de $18,9 \mathrm{dBm}$ à la fréquence de $2 \mathrm{GHz}$.

Le facteur de bruit au-delà de $200 \mathrm{MHz}$ est en moyenne de 5,5 dB. 


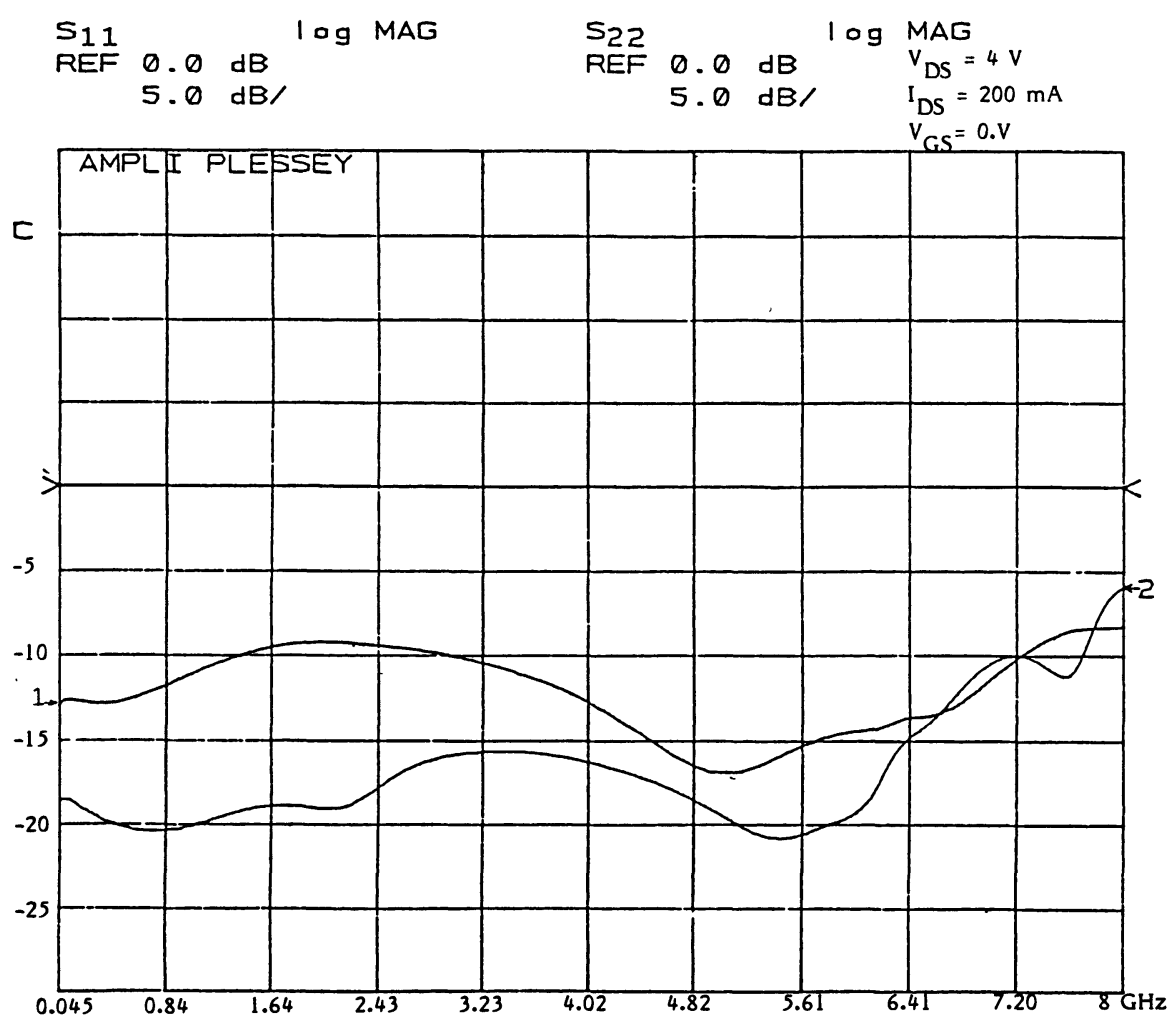

Fig. 10. - Courbes de TOS entrée et sortie de l'amplificateur monolithique.

[VSWR in and VSWR out of monolithic amplifier.]

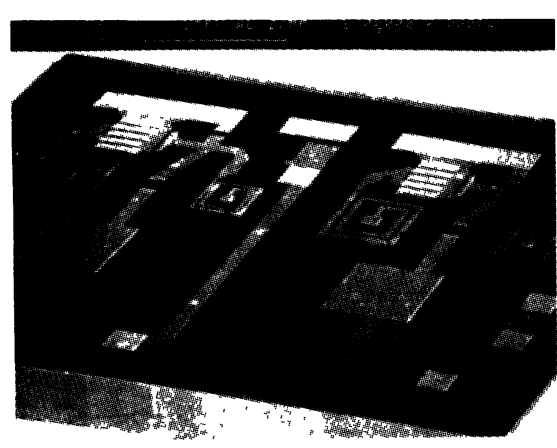

Fig. 11. - Réalisation monolithique.

[Monolithic realization.]

Le tableau suivant donne les principales caractéristiques des deux amplificateurs, hybride et monolithique.

\begin{tabular}{|l|c|c|c|c|}
\hline & Gain & $\begin{array}{c}\text { Nombre } \\
\text { d'étages }\end{array}$ & $\begin{array}{c}\text { Facteur } \\
\text { de bruit }\end{array}$ & $\begin{array}{c}\text { Puissance } \\
\text { de sortie }\end{array}$ \\
\hline Hybride & $15,5 \mathrm{~dB}$ & 3 & $9,5 \mathrm{~dB}$ & $9 \mathrm{dBm}$ \\
\hline Monolithique & $17 \mathrm{~dB}$ & 2 & $5,5 \mathrm{~dB}$ & $18,9 \mathrm{dBm}$ \\
\hline
\end{tabular}

\section{Conclusion.}

Cette procédure simple de synthèse des circuits permet donc de concevoir et de réaliser des amplificateurs à plusieurs étages. La méthode des fréquences réelles est basée uniquement sur la connaissance des paramètres de répartition des transistors et ne nécessite aucun modèle ou schéma équivalent. Elle tient compte de la non-unilatéralité des transistors mais aussi des impératifs technologiques (polarisation, découplage, encombrement). Les réalisations en technologies hybride et monolithique ont confirmé les résultats théoriques, en particulier en monolithique où l'on disposait d'un transistor plus performant et de modèles plus précis pour les éléments passifs.

La méthode des fréquences réelles, et le logiciel FREEL peuvent être étendus à l'étude et l'optimisation du bruit ainsi qu'à la conception d'amplificateurs de puissance présentant une structure arborescente. Il est également possible d'optimiser les égaliseurs directement en éléments distribués.

\section{Remerciements.}

Les auteurs tiennent à remercier MM. P. Legaud, R. Boitin du CNET Lannion B ROC pour les mesures des réponses temporelles des amplificateurs ainsi que J. Le Bihan de l'Université de Brest pour son aide tout au long de l'étude. 


\section{Bibliographie}

[1] Carlin H. J., A New Approach to gain-bandwidth problems, IEEE Trans. CAS 23 (1987) 170.

[2] YARMAN B. S., A Simplified «Real Frequency» Technique Applied to broad-band multistage microwave amplifier, IEEE Trans. MTT 30 (1982) 2216-2222.

[3] Belevitch V., Elementary application to the scattering formalism to network design, IRE Trans. Cire Theo CT 3 (juin 1956).

[4] Jarry P., Perennec A., Optimization of Gain and VSWR in multistage microwave amplifiers using the real frequency method, Invited paper, ECCTD'87 Paris (septembre 1987) pp. 203-208$1-4$.
[5] Jarry P., Perennec A., Le Bihan J., Optimisation et synthèse d'amplificateurs microondes par la méthode des fréquences réelles, Revue Phys. Appl. 23 (1988) 137-142.

[6] Perennec A., Soares R., Jarry P., Legaud P., BoITTIN R., Application of circuit synthesis to the design of a three-stage, $4 \mathrm{MHz}$ to $7 \mathrm{MHz}$ Amplifier for a wide band fiber optics receiver, EMC septembre 1988 (Stockholm).

[7] Perennec A., Conception et réalisation d'amplificateurs microondes par la méthode des fréquences réelles, Thèse de doctorat nouveau régime, juillet 1988 (Université de Brest).

[8] SAF, Design Manfred Platz, Marienstrasse $17 \mathrm{~b}$ 1000 Berlin, 4 S West Germany. 